\title{
Underlying Processes driving the Evolution of Nanoporous Silica in Water and Electrolyte Solutions
}

\author{
Markus Baum * ${ }^{\circ}$, Francois Rieutord $t^{\circ}$, Diane Rébiscoul ${ }^{* 0} \oint$ \\ * CEA, ICSM - UMR 5257 CEA-CNRS-UM-ENSCM, 30207 Bagnols-sur-Cèze Cedex, France \\ $\$$ IRIG - CEA-Grenoble, Interdisciplinary Research Institute of Grenoble (IRIG), 17 avenue des Martyrs 38054 \\ Grenoble Cedex 09 \\ Grenoble, FR 38054 \\ ${ }^{\circ} \mathrm{PhD}$ title
}

$\S$ Corresponding author: diane.rebiscoul@cea.fr

\section{This PDF file includes:}

- Supplementary text

- $\quad$ Figures S1 to S6

- Tables S1

- SAXS pattern (scheme depicting the parameters in Table 1), pore size distributions and nitrogen adsorptiondesorption isotherms of MCM-41 and SBA-15

- $\mathrm{pH}$ values of the electrolyte solutions

- Decrease of the Bragg peaks in the experimental SWAXS patterns

- Comparison between calculated SAXS pattern and the experimental spectrum of MCM-41

- Method to calculate the SAXS patterns of SBA-15

- Calculation of $R_{a l}$ and $D_{\text {micro }}$

- $\quad$ SI References

\section{Supplementary information Text}

\section{MCM-41 and SBA-15 silica materials}

Adsorption-desorption isotherms of silica materials are presented in Figure S1 (a). The curves for both materials correspond to typical type IV (IUPAC) isotherms whereas adsorption-desorption of SBA-15 exhibits a hysteresis of type H1. This hysteresis loop is associated with capillary condensation in mesoporous structures. In this 
case, the parallel desorption and adsorption branches are related to a narrow pore size distribution. From these isotherms, the characteristics of the mesoporous materials (specific surface areas from the Brunauer-Emmett-Teller (BET) model SBET, pore volumes Vp and pore size distributions from the Barret-Joyner-Halenda model presented Figure S1 (b)) were determined and are presented in Table 1.
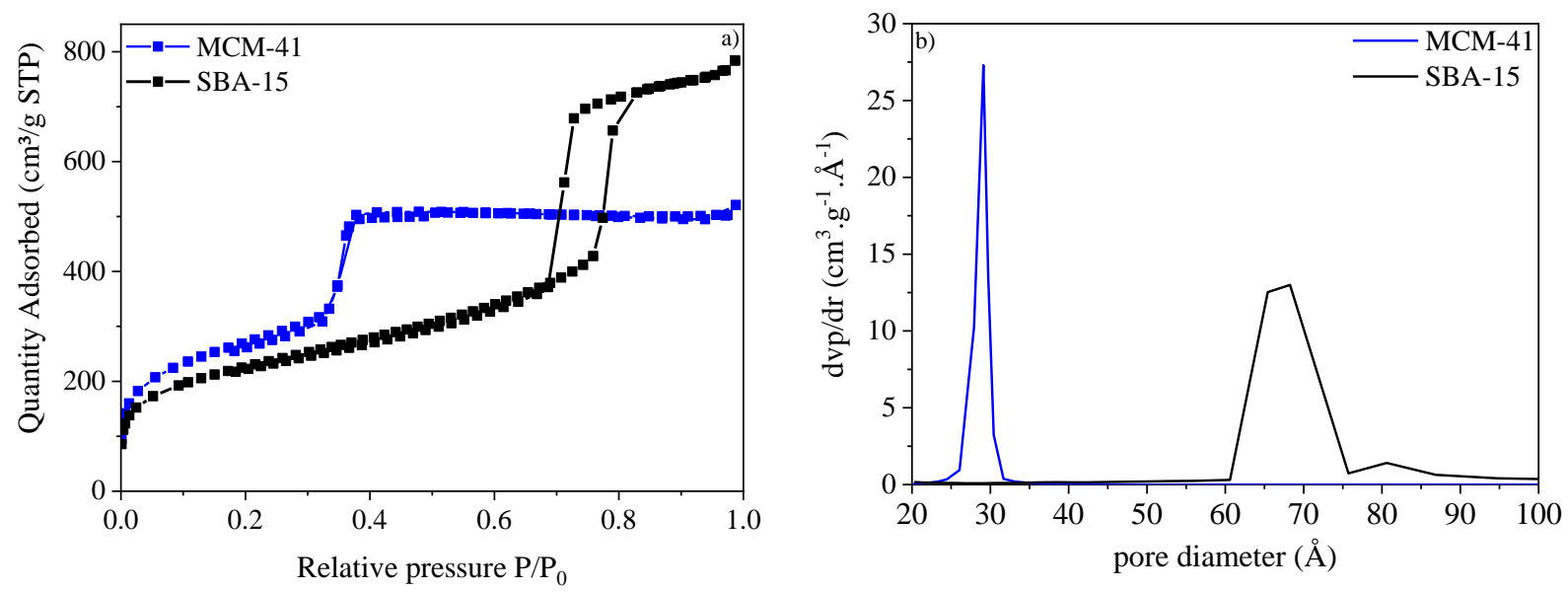

Figure S1: (a) Nitrogen adsorption-desorption isotherms and (b) pore size distributions of SBA-15 and MCM-41 used in this study.

Figure S2 presents the SAXS patterns of SBA-15 and MCM-41. The patterns of SBA-15 presents a strong and well-defined peak at $\mathrm{q}=0.67 \mathrm{~nm}^{-1}$ and two peaks of weaker intensities at around $1.15 \mathrm{~nm}^{-1}$ and $1.33 \mathrm{~nm}^{-1} . \mathrm{Since}$ MCM-41 has a smaller confinement than SBA-15, Bragg peaks are shifted to higher $\mathrm{q}$ values $\left(\mathrm{q}_{1}=1.58 \mathrm{~nm}^{-1}, \mathrm{q}_{2}=\right.$ $2.72 \mathrm{~nm}^{-1}$ and $\left.\mathrm{q}_{3}=3.10 \mathrm{~nm}^{-1}\right)$. These peaks correspond to the $(10),(1 \overline{1})$ and (20) lattice planes of the hexagonal pore arrangement. From the experimental data, inter-reticular distances $d$ and the unit cell parameter a $a_{0}$ were calculated by using eqs. [1] and [2].

$$
d=\frac{\lambda}{2 \sin \theta}
$$

where $\lambda$ is the wavelength of incident wave; $d$ is the spacing between the planes in the mesoporous lattice; and $\theta$ is the angle between the incident beam and the scattering planes. The unit cell parameter $a_{0}$ was also deduced from eq. [1] and [2]:

$$
a_{0}=\frac{2 d_{100}}{\sqrt{3}}
$$


It is important to note here that only SBA-15 presents a microporosity resulting from the penetration of PEO surfactant chains into the silica wall during its synthesis (1-3). Mean pore sizes were deduced from the pore size distribution obtained from the BJH model (Figure S1 (b)).
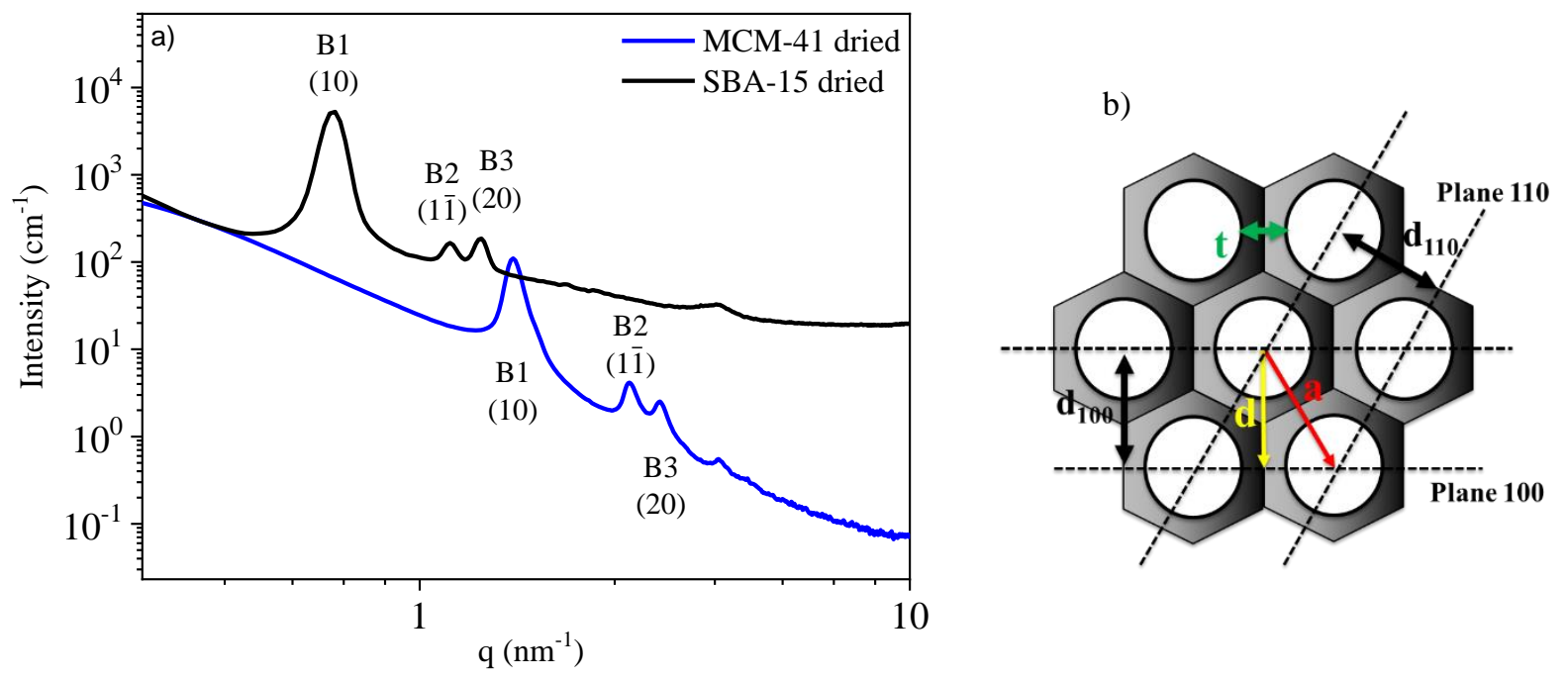

Figure S2: Small-Angle X-Ray Scattering spectra of MCM-41 and SBA-15. The right panel presents the various parameters defining the hexagonal arrangement of the mesopores.

\section{pH of the electrolyte solutions}

The final $\mathrm{pH}$ values of the electrolyte solutions $\mathrm{XCl}_{2}$ (with $\mathrm{X}=\mathrm{Ba}^{2+}, \mathrm{Ca}^{2+}$ and $\mathrm{Mg}^{2+}$ ) measured at the laboratory atmosphere a re presented in Table S1.

Table S1: $\mathrm{pH}$ values of electrolyte solutions $\mathrm{XCl}_{2}$ (with $\mathrm{X}=\mathrm{Ba}^{2+}, \mathrm{Ca}^{2+}$ and $\mathrm{Mg}^{2+}$ ) measured at laboratory atmosphere. The uncertainties do not exceed \pm 0.05 .

\begin{tabular}{cccc}
\hline$\left[\mathrm{XCl}_{2}\right]_{0}$ & $0.01 \mathrm{M}$ & $0.2 \mathrm{M}$ & $1 \mathrm{M}$ \\
\hline \hline $\mathrm{BaCl}_{2}$ & 5.79 & 5.47 & 5.38 \\
$\mathrm{CaCl}_{2}$ & 6.22 & 6.31 & 6.37 \\
$\mathrm{MgCl}_{2}$ & 6.35 & 6.07 & 5.77 \\
\hline
\end{tabular}




\section{Comparison between calculated SAXS pattern and the experimental spectrum of MCM-41}
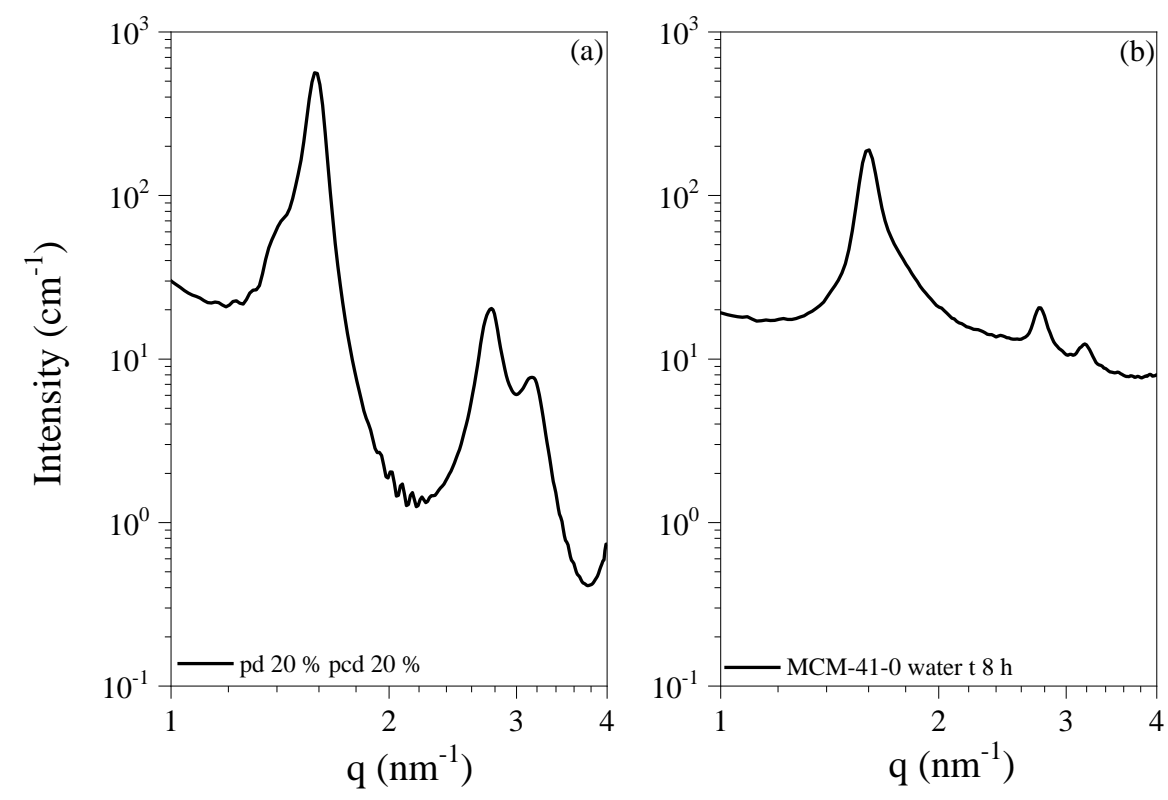

Figure S3: Comparison between calculated SAXS pattern (a) of a mesoporous lattice with a pore radius of $1.8 \mathrm{~nm}$ and unit cell parameter of $4.6 \mathrm{~nm}$ and the experimental spectrum of MCM-41 at the beginning of the alteration experiment (b).

As shown in Figure S3, the best compromise between the absolute intensity level adjustment and the overall shape of the diffraction pattern is obtained considering a porous lattice parameter of $4.6 \mathrm{~nm}$, with a paracrystallinity degree of $20 \%$ and a mean pore radius of $1.8 \mathrm{~nm}$. It is also important to note that a pore radius distribution of $20 \%$ of the mean pore radius was introduced to the model by considering it to have a Gaussian shape. The intensity ratio between the experimental and simulated Bragg peaks are well reproduced. However, the absolute intensities may differ since no additional background was added to the simulated patterns. 


\section{Decrease of the Bragg peaks in the experimental SWAXS patterns}

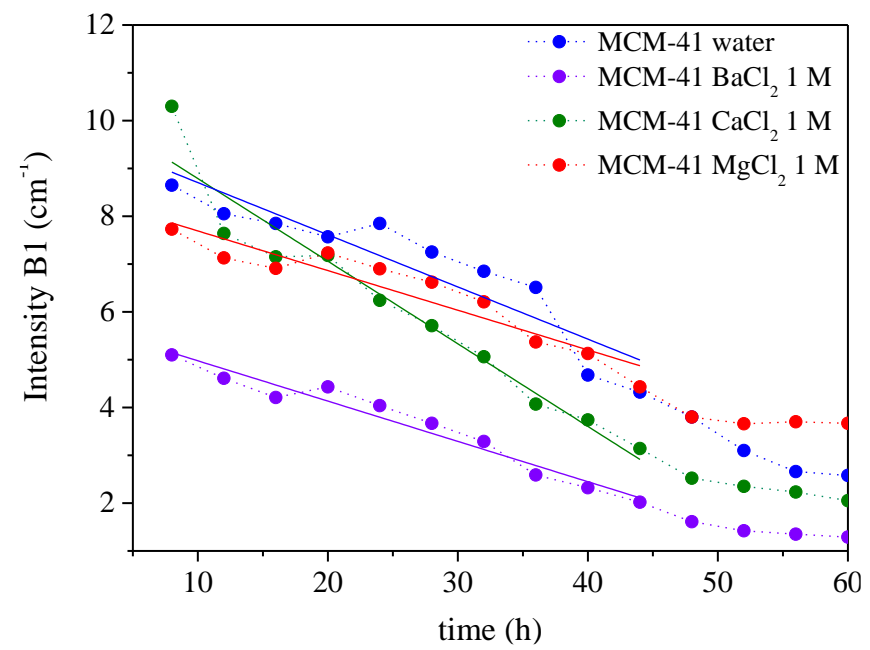

Figure S4: Evolutions of the intensities and linear fits of the Bragg peaks B1 of the SWAXS patterns of the MCM-41 in water and electrolyte solutions as a function of the alteration time.

\section{Method to calculate the SAXS patterns of SBA-15}

The hexagonal arrangement of cylindrical pores of infinite (for the sake of convenience) length are recreated by three main parameters: pore size $r_{p}$, wall thickness $w$ and unit cell parameter $a$. It is important to note that compared to MCM-41, the paracrystalline disorder parameter and the Gaussian distribution for the pore diameters are kept constant for SBA-15. The density of silica was chosen to $d_{\mathrm{SiO} 2}=2.2 \mathrm{~g} \cdot \mathrm{cm}^{-3}$. Furthermore, outside the mesopores, a supplementary layer was taken into account having a different density $\left(\mathrm{d}_{\mathrm{SiO} 2 \text {, microporous }}=1.68 \mathrm{~g} \cdot \mathrm{cm}^{-3}\right)$ and a thickness alw. This layer represents the microporous alteration layer around the mesopores. Moreover, the electron densities of electrolyte solutions used to fill the pores were considered and are summarized in Table S2. Different SAXS spectra were calculated by changing the pore size, the thickness of the alteration layer and the electron density of the various electrolyte solutions. From the calculated curves, the intensities of Bragg peaks B2 and B3, I2 and I3, as well as the ratio I2/I3, were determined. For one modelled I2/I3 ratio, between 1 and 4 couples rp (pore size) and alw (alteration layer thickness) were obtained. To determine the most coherent couple, we have considered that the evolution of I2 and I3 obtained from calculations should be similar to the experimental ones. From this condition, only one couple rp and alw was possible. From the comparison, it was possible to quantify the time-dependent evolution of the pore morphology and the alteration layer. 
Table S2. Mass densities $\rho_{\mathrm{m}}$, and electron densities $\rho$ el of water and electrolyte solutions $\mathrm{XCl}_{2}(\mathrm{X}=$ Ba, Ca, Mg).

\begin{tabular}{ccc}
\hline solution & $\begin{array}{c}\rho_{\mathrm{m}} \\
\left(\mathrm{g} . \mathrm{L}^{-1}\right)\end{array}$ & $\begin{array}{c}\rho_{\mathrm{el}} \\
\left(\mathrm{e} . \AA^{-3}\right)\end{array}$ \\
\hline water & 1.0 & 0.334 \\
$\mathrm{BaCl}_{2} 1 \mathrm{M}$ & 1.178 & 0.379 \\
$\mathrm{CaCl}_{2} 1 \mathrm{M}$ & 1.077 & 0.356 \\
$\mathrm{MgCl}_{2} 1 \mathrm{M}$ & 1.084 & 0.358
\end{tabular}

\section{Calculation of $\mathbf{R}_{\text {al }}$}

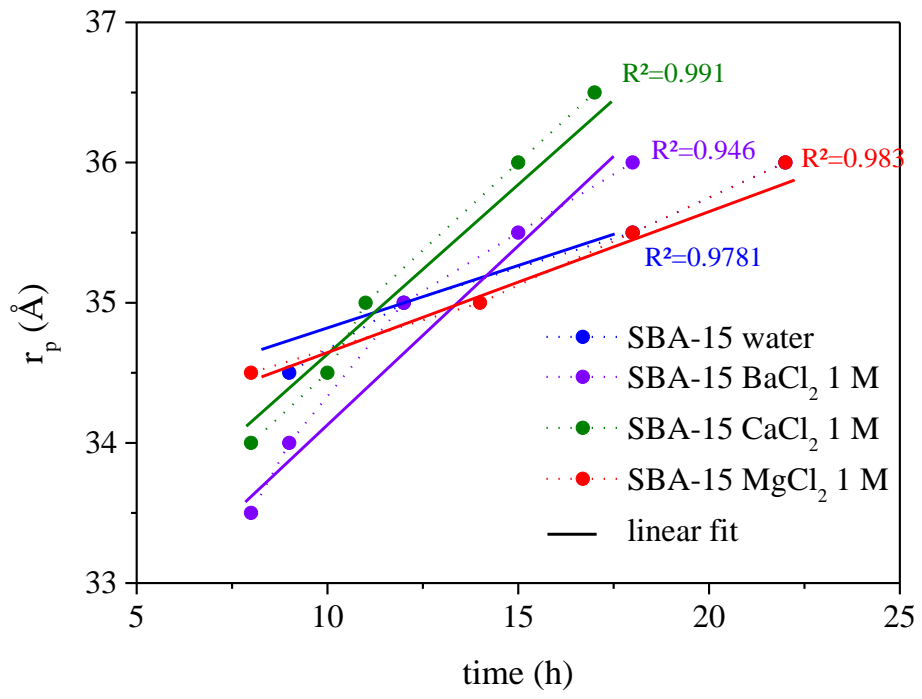

Figure S5: Evolutions of $r_{p}$ of SBA-15 as function of alteration time and linear fits used for the calculation of $R_{a l}$ in water and electrolyte solutions. 


\section{Calculation of $\mathbf{D}_{\text {micro }}$}

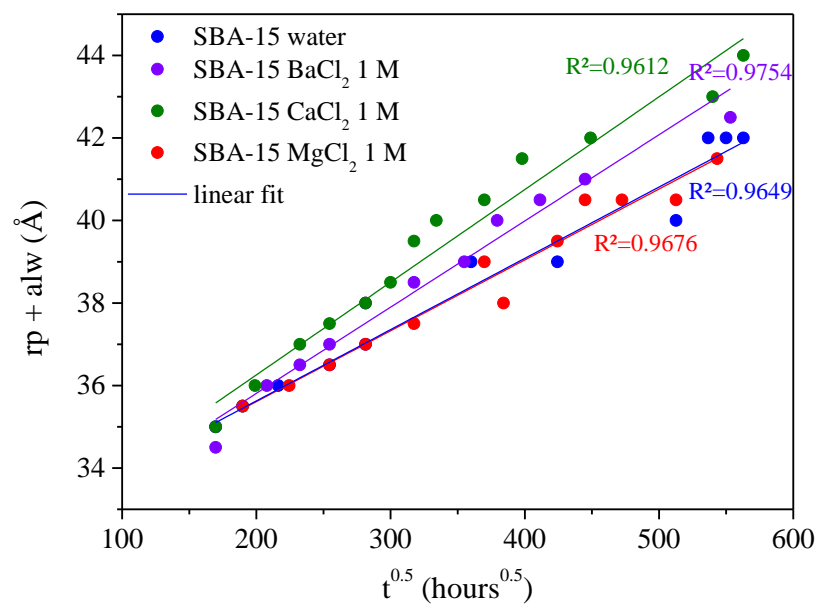

Figure S6: Evolutions of $r_{p}+$ alw with the square root of time and linear fits used to calculate $D_{\text {micro. }}$.

\section{References}

1. M. Templin, et al. (1997), Organically modified aluminosilicate mesostructures from block copolymer phases. Sci. 278, 1795-8.

2. D. Zhao, et al. (1998), Triblock copolymer syntheses of mesoporous silica with periodic 50 to 300 angstrom pores. Science 279, 548-52.

3. N. A. Melosh, et al. (1999), Molecular and Mesoscopic Structures of Transparent Block Copolymer-Silica Monoliths. Macromolecules 32, 4332-4342. 